\title{
Screening of Chilli Genotypes for Resistance to Fruit Rot caused by Colletotrichum capsici at Fruit Bearing and Harvest Stages
}

\author{
M. N. Sharath ${ }^{1 *}$, R. K. Mesta ${ }^{1}$, M. A. Kareem ${ }^{2}$, P. S. Ajjappalavara ${ }^{2}$ and Ambresh ${ }^{2}$ \\ ${ }^{1}$ Department of plant pathology, College of Horticulture Bagalkote, India \\ ${ }^{2}$ Department of plant pathology, Horticultural research and extension center, Devihosur, \\ University of horticultural sciences Bagalkote 587 104, India \\ *Corresponding author
}

\section{A B S T R A C T}

\begin{tabular}{|l|}
\hline K e y w o r d s \\
Chilli, \\
$\begin{array}{l}\text { Colletotrichum } \\
\text { capsici, Screening } \\
\text { and Resistant. }\end{array}$ \\
\hline Article Info \\
\hline $\begin{array}{l}\text { Accepted: } \\
\text { 10 July } 2020 \\
\text { Available Online: } \\
\text { 10 August } 2020\end{array}$ \\
\hline
\end{tabular}

The field and laboratory screening of 31 accessions were carried out against fruit rot of chilli caused by Colletotrichum capsici under natural pressure condition. Out of 31 accessions eight were found resistant, remaining twenty three accessions found moderately resistant at 90 days after transplanting. The reaction during harvest stages varied from that of 90 days after harvest which recorded, 2 resistant accessions, eight moderately resistant accessions, twenty moderately susceptible accessions and Byadagi Dabbi as susceptible. During in vitro screening 18 accessions were found resistant, 8 were moderately resistant, 3 were moderately susceptible and 2 were susceptible to the pathogen during green stage of the fruit. The reaction of chilli fruits during turning red stage recorded, 18 accessions as moderately resistant, 8 as moderately susceptible and one (DCA-298) was susceptible. The reaction varied in red stage of fruit development wherein, 5 accessions were resistant, 18 accessions were moderately resistant, 6 were moderately susceptible and 2 were susceptible to the pathogen. The accessions DCA-67, 69, 189, 302 and 305 were reacted resistant to the $C$. capsici in field and artificial screening. Hence, these accessions having the resistant genes which can be used for resistant breeding programme.

\section{Introduction}

Chilli (Capsicum annuum) is an important vegetable as well asspice crop belongs to Solanaceae family. The genus Capsicum was assumed to have been originated from Mexico, Central America and South America (Perry et al., 2007).Five species of Capsicum (C. annuum, C. baccatum, C. chinense, $C$. frutescens and $C$. pubescens) are commonly domesticated and cultivated in different parts of the world. Among these five species, $C$. annuum is the most commonly cultivated worldwide, followed by $C$. frutescens (Bosland, 1994). Chilli is used in all forms starting from fresh green fruits, ripe fruits, dried fruits and powdered form. Fresh green pungent fruits are generally used in salads, stuffing and as a flavoring agent in cooked meals. Indian chilli is considered to be world famous for two important commercial qualities viz., color and pungency levels. 
Indian chilli is mainly exported to Asian countries like Vietnam, Thailand, Sri Lanka, Bangladesh and U.A.E. In India, Andhra Pradesh, Karnataka, Telangana, Uttar Pradesh, Bihar and Madhya Pradesh are major chilli growing states. In Karnataka, greenchilli is grown over an area of 45,910 ha with the production of $6,73,810$ MT while dry chilliis grown over 1, 27, 600 ha with the production of 2, 60, 140 MT (Anonymous, 2018). Naturally, the chilli production were affected by both biotic, such as microorganisms (plant pathogenic fungi, bacteria, nematodes, viruses), insect pests (aphids, thrips, mites, midges and fruit borer), weeds and abiotic factors, such as temperature, moisture, light and chemicals. The anthracnose disease caused by Colletotrichum capsici is one of the major and devastating diseases of chilli, which causes 10-60 Per cent yield loss apart from reducing quality. This disease was first time reported in India by Sydow during 1913 from Coimbatore (Ridzuan et al., 2018).The present study was carried out to evaluate disease resistance reaction of agronomically superior chilli accessions.

\section{Materials and Methods}

\section{Screening of chilli accessions against fruit rot under natural condition}

Screening of chilli accessions for resistance against fruit rot was conducted at Horticulture Research and Extension Center, Haveri (Devihosur), during kharif2018-19.

The accessions used for the experiment were selected from the germplasm pool of Horticultural Research and Extension Center (HREC), Haveri (Devihosur) which were found superior in yield and other desirable characters. Screening of 31 chilli accessions for resistance to fruit rot caused by $C$. capsici was carried out in the field at HREC, Devihosur, both under natural and artificial inoculation of pathogen. Total 31accessions were planted $5 \mathrm{~m}$ single lines with two replications with a spacing of $60 \times 60 \mathrm{~cm}$. Sowing works were carried out during last week of June 2018 and transplanting were carried out during last week of July 2018.In field condition, accessions were screened against $C$. capsici at 90 days after transplanting and during harvest.

In vitro screening of chilli accessions for resistance to fruit rot caused by Colletotrichum capsici by challenge inoculation of the pathogen to detached fruits

Spores of $C$. capsici were harvested by adding sterile distilled water to the well sporulated culture plates containing $C$. capsici and diluted the conidial suspension by adding 100 $\mathrm{ml}$ of sterile distilled water. The spore load was counted using haemocytometer.

Fruits from each accession at green stage, turning green to red stage and red ripe stage are bought to the laboratory and were surface sterilized with 70 per cent ethanol and washed with sterile distilled water. Three fruits were inoculated with the pathogen inoculum, which was harvested from the $C$. capsici culture plates. The inoculation was done through sterile micro syringe at a concentration of 2.4 $\mathrm{x} 10^{5}$, one fruit was kept as control, where the sterile distilled water without spore was injected. These fruits were placed in Petri plates containing moist tissue paper to maintain the humidity inside the Petri plates. Observations on disease severity were taken at 1 and 3 days after inoculation. The screening of accessions was done by scoring of fruits using standard scale.

Disease intensity was measured by grading the fruits using 0-9 scale given by Mayee and Datar (1986). The scale is as follows. 


\begin{tabular}{|l|l|l|}
\hline Grade & $\begin{array}{l}\text { Per cent fruit } \\
\text { infection }\end{array}$ & Disease reaction \\
\hline $\mathbf{0}$ & 0 & Immune \\
\hline $\mathbf{1}$ & $1-10$ & Resistant \\
\hline $\mathbf{3}$ & $11-25$ & Moderately resistant \\
\hline $\mathbf{5}$ & $26-50$ & $\begin{array}{l}\text { Moderately } \\
\text { susceptible }\end{array}$ \\
\hline $\mathbf{7}$ & $51-75$ & Susceptible \\
\hline $\mathbf{9}$ & $>75$ & Highly susceptible \\
\hline
\end{tabular}

The per cent disease index (PDI) was calculated to record the disease intensity, according to the following formula given by Wheeler (1969).

Per cent disease index $(\mathrm{PDI})=\frac{\text { Sum of all numerical }}{\text { Number of fruits } x \text { Maximum observed disease grade }} \times 100$

\section{Results and Discussion}

Screening of chilli accessions against fruit rot under natural condition

The fruits were graded as per scale and percent disease index was recorded at 90 days after transplanting and during harvesting stage. Data are presented in table 1

During 90 DAT, none of the 31 accessions showed immune reaction, 8 accessions (DCA $67,69,76,189,237,257,302$ and 305) were found resistant and other 23accessions were found moderately resistant to the pathogen. The least PDI was recorded in DCA- 69 (4.45) which was on par with DCA- 305 (5.78) and DCA- 237 (8.89) and highest was noticed in DCA- 145 (24.00) which was on par with DCA- 76 (23.33) and DCA- 176 (20.89).

During harvest, none of the 31 accessions was immune against the pathogen. Among 31 accessions, 2 accessions (DCA-69 and 189) was resistant, 8 accessions (DCA- 67, 76, 89, $123,237,300,302$ and 305) were categorized under moderately resistant whereas, 20 were moderately susceptible to the pathogen and Byadagi Dabbi categorized under susceptible. The least PDI was recorded in DCA- 69 and DCA-189(10.22) followed by DCA- 302 (12.44) and DCA- 305 (18.22) and highest PDI was recorded in Byadagi Dabbi (39.78) and DCA- 216 (39.78) followed by DCA- 234 (37.11).

In vitro screening of chilli accessions for resistance to fruit rot caused by Colletotrichum capsici by challenge inoculation of the pathogen to detached fruits

The observations on disease severity were recorded during 1 and 3 day after inoculation. The results are presented in table 2 .

Among 31accessions, 24accessions showed immune without any lesions on the fruits, 7accessions (DCA-76, 107, 145, 222, 234, 240 and 298) developed lesions on the fruits during 1 day after inoculation (DAI) in green stage. But, the lesion diameter and length increased during $3 \mathrm{DAI}$, the PDI varied from 3.70 to 62.96 (DCA- 145).During 3 days after inoculation at green stage, 18accessionsviz., DCA-67, 69, 76, 79, 81, 89, 104, 118, 123, $153,174,189,216,224,233,237,300$ and 305 were found resistant, 8 accessions (DCA98, 154, 176, 222, 240, 257, 295 and Byadagi Dabbi) were moderately resistant, 2accessions (DCA-234 and 302) were moderately susceptible and 3 accessions (DCA-107, 145 and 298) were susceptible (Figure 1).

Among 31accessions, 12accessions reacted as immune and 19accessions developed the lesions after 1 day of inoculation during turning green to red stage. After 3 days of inoculation the PDI varied from 7.41 to 55.56 (DCA- 298). During 3 days after inoculation at turning green to red stage, 4 accessions (DCA-69, 89, 145 and 189) were found resistant, 18 accessions were moderately 
resistant, 8 accessions (DCA- 81, 98, 118, 174, 216, 233, 234 and 237) were moderately susceptible and DCA-298was susceptible to the pathogen.

About 16accessions were reacted as immune to the pathogen and 15accessions developed lesions during red ripe stage. After 3 days of inoculation the PDI was recorded varied from 3.70 (DCA- 69) to 55.56 (DCA- 174). During 3 days after inoculation at red ripe stage, 5 accessions (DCA-67, 69, 89, 189 and 302) were found resistant, 18 were moderately resistant, 6 accessions (DCA-79, 81, 233, 237, 257 and 300) were moderately susceptible and DCA- 174 and Byadagi Dabbi were susceptible to the pathogen (Figure 2).

The reaction of different entries of chilli accessions to $C$. capsici infection at field screening, challenge inoculation and in vitro screening are depicted in table 3.
The field screening of 31accessions was carried out under natural pressure, out of which only eight accessions were found resistant and remaining twenty three accessions were found to be moderately resistant at 90 days after transplanting. The reaction during harvest stages varied from that of 90 days after harvest which recorded, 2 accessions as resistant, eight accessions as moderately resistant, twenty accessions were moderately susceptible and ByadagiDabbi as susceptible. Koppad (2014) screened 250 accessions of chilli and found that none of them was immune to $C$. capsici while, 43 accessions were resistant and 110 were moderately resistant for fruit rot under field condition. Similar results were reported by Ruth et al., (2007), Kaur and Singh (2009), Singh and Chowdhury (2011), Varma et al., (2012) and Prasath and Ponnuswami (2008).

Table.1 Screening of chilli accessions under natural field conditions against fruit rot during kharif-2018

\begin{tabular}{|c|c|c|c|c|c|c|c|}
\hline Sl. No. & Genotypes & $\begin{array}{l}90 \text { days after } \\
\text { transplanting } \\
\text { (PDI) }\end{array}$ & $\begin{array}{l}\text { Max. } \\
\text { grade }\end{array}$ & Reaction & $\begin{array}{l}\text { During harvest } \\
\text { (PDI) }\end{array}$ & $\begin{array}{l}\text { Max. } \\
\text { grade }\end{array}$ & Reaction \\
\hline 1 & DCA 67 & $\begin{array}{c}10.22 \\
(18.62)\end{array}$ & 1 & $\mathrm{R}$ & $\begin{array}{c}27.78 \\
(31.79)\end{array}$ & 3 & MR \\
\hline 2 & DCA 69 & $\begin{array}{c}4.45 \\
(12.15)\end{array}$ & 1 & $\mathrm{R}$ & $\begin{array}{c}10.22 \\
(18.62)\end{array}$ & 1 & $\mathrm{R}$ \\
\hline 3 & DCA 76 & $\begin{array}{c}23.33 \\
(28.87)\end{array}$ & 1 & $\mathrm{R}$ & $\begin{array}{c}23.11 \\
(28.72)\end{array}$ & 3 & MR \\
\hline 4 & DCA 79 & $\begin{array}{c}16.22 \\
(23.73)\end{array}$ & 3 & MR & $\begin{array}{c}25.56 \\
(30.35)\end{array}$ & 5 & MS \\
\hline 5 & DCA 81 & $\begin{array}{c}16.00 \\
(23.55)\end{array}$ & 3 & MR & $\begin{array}{c}23.78 \\
(29.17)\end{array}$ & 5 & MS \\
\hline 6 & DCA 89 & $\begin{array}{c}12.00 \\
(20.25)\end{array}$ & 3 & MR & $\begin{array}{c}22.67 \\
(28.42)\end{array}$ & 3 & MR \\
\hline 7 & DCA 98 & $\begin{array}{c}17.56 \\
(24.75)\end{array}$ & 3 & MR & $\begin{array}{c}28.23 \\
(32.08)\end{array}$ & 5 & MS \\
\hline 8 & DCA 104 & $\begin{array}{c}18.67 \\
(25.58)\end{array}$ & 3 & MR & $\begin{array}{c}26.67 \\
(31.08)\end{array}$ & 5 & MS \\
\hline 9 & DCA 107 & $\begin{array}{c}18.22 \\
(25.25)\end{array}$ & 3 & MR & $\begin{array}{c}32.67 \\
(34.84)\end{array}$ & 5 & MS \\
\hline
\end{tabular}




\begin{tabular}{|c|c|c|c|c|c|c|c|}
\hline 10 & DCA 118 & $\begin{array}{c}12.00 \\
(20.25)\end{array}$ & 3 & MR & $\begin{array}{c}33.33 \\
(35.25)\end{array}$ & 5 & MS \\
\hline 11 & DCA 123 & $\begin{array}{c}14.22 \\
(22.11)\end{array}$ & 3 & MR & $\begin{array}{c}19.34 \\
(26.07)\end{array}$ & 3 & MR \\
\hline 12 & DCA 145 & $\begin{array}{c}24.00 \\
(29.32)\end{array}$ & 3 & MR & $\begin{array}{c}30.45 \\
(33.47)\end{array}$ & 5 & MS \\
\hline 13 & DCA 153 & $\begin{array}{c}15.78 \\
(23.38)\end{array}$ & 3 & MR & $\begin{array}{c}18.67 \\
(25.58)\end{array}$ & 5 & MS \\
\hline 14 & DCA 154 & $\begin{array}{c}20.45 \\
(26.87)\end{array}$ & 3 & MR & $\begin{array}{c}36.00 \\
(36.86)\end{array}$ & 5 & MS \\
\hline 15 & DCA 174 & $\begin{array}{c}19.11 \\
(25.91)\end{array}$ & 3 & MR & $\begin{array}{c}31.78 \\
(34.30)\end{array}$ & 5 & MS \\
\hline 16 & DCA 176 & $\begin{array}{c}20.89 \\
(27.17)\end{array}$ & 3 & MR & $\begin{array}{c}36.23 \\
(36.99)\end{array}$ & 5 & MS \\
\hline 17 & DCA 189 & $\begin{array}{c}10.45 \\
(18.84)\end{array}$ & 1 & $\mathrm{R}$ & $\begin{array}{c}10.22 \\
(18.62)\end{array}$ & 1 & $\mathrm{R}$ \\
\hline 18 & DCA 216 & $\begin{array}{c}20.00 \\
(26.55)\end{array}$ & 3 & MR & $\begin{array}{c}39.78 \\
(39.08)\end{array}$ & 5 & MS \\
\hline 19 & DCA 222 & $\begin{array}{c}17.56 \\
(24.76)\end{array}$ & 3 & MR & $\begin{array}{c}31.33 \\
(34.02)\end{array}$ & 5 & MS \\
\hline 20 & DCA 224 & $\begin{array}{c}16.67 \\
(24.07)\end{array}$ & 3 & MR & $\begin{array}{c}32.00 \\
(34.43)\end{array}$ & 5 & MS \\
\hline 21 & DCA 233 & $\begin{array}{c}19.33 \\
(26.07)\end{array}$ & 3 & MR & $\begin{array}{c}32.23 \\
(34.57)\end{array}$ & 5 & MS \\
\hline 22 & DCA 234 & $\begin{array}{c}18.45 \\
(25.42)\end{array}$ & 3 & MR & $\begin{array}{c}37.11 \\
(37.51)\end{array}$ & 5 & MS \\
\hline 23 & DCA 237 & $\begin{array}{c}8.89 \\
(17.32)\end{array}$ & 1 & $\mathrm{R}$ & $\begin{array}{c}25.12 \\
(30.05)\end{array}$ & 3 & MR \\
\hline 24 & DCA 240 & $\begin{array}{c}12.44 \\
(20.64)\end{array}$ & 3 & MR & $\begin{array}{c}35.34 \\
(36.45)\end{array}$ & 5 & MS \\
\hline 25 & DCA 257 & $\begin{array}{c}10.00 \\
(18.42)\end{array}$ & 1 & $\mathrm{R}$ & $\begin{array}{c}26.89 \\
(31.22)\end{array}$ & 5 & MS \\
\hline 26 & DCA 295 & $\begin{array}{c}20.00 \\
(26.55)\end{array}$ & 3 & MR & $\begin{array}{c}25.56 \\
(30.35)\end{array}$ & 5 & MS \\
\hline 27 & DCA 298 & $\begin{array}{c}14.00 \\
(21.96)\end{array}$ & 3 & MR & $\begin{array}{c}29.33 \\
(32.78)\end{array}$ & 5 & MS \\
\hline 28 & DCA 300 & $\begin{array}{c}9.56 \\
(17.97)\end{array}$ & 3 & MR & $\begin{array}{c}23.34 \\
(28.86)\end{array}$ & 3 & MR \\
\hline 29 & DCA 302 & $\begin{array}{c}10.00 \\
(18.42)\end{array}$ & 1 & $\mathrm{R}$ & $\begin{array}{c}12.44 \\
(20.64)\end{array}$ & 3 & MR \\
\hline 30 & DCA 305 & $\begin{array}{c}5.78 \\
(13.86)\end{array}$ & 1 & $\mathrm{R}$ & $\begin{array}{c}18.22 \\
(25.23)\end{array}$ & 3 & MR \\
\hline \multirow[t]{3}{*}{31} & ByadagiDabbi & $\begin{array}{c}15.78 \\
(23.38)\end{array}$ & 3 & MR & $\begin{array}{c}59.78 \\
(61.08)\end{array}$ & 7 & S \\
\hline & $\mathrm{SE}(\mathrm{m})$ & 0.83 & & & 0.69 & & \\
\hline & C.D. & 2.42 & & & 2.00 & & \\
\hline
\end{tabular}


Int.J.Curr.Microbiol.App.Sci (2020) 9(8): 142-152

Table.2 In vitro screening of chilli accessions against $C$. capsici using microinjection method

\begin{tabular}{|c|c|c|c|c|c|c|c|c|c|c|}
\hline \multirow[t]{2}{*}{ Sl. No } & \multirow[t]{2}{*}{ Genotypes } & \multicolumn{3}{|c|}{ Green stage } & \multicolumn{3}{|c|}{ Turning red stage } & \multicolumn{3}{|c|}{ Red ripe stage } \\
\hline & & 1DAI & 3DAI & Reaction & 1DAI & 3DAI & Reaction & 1DAI & 3DAI & Reaction \\
\hline 1 & DCA 67 & 0.00 & 3.70 & $\mathrm{R}$ & 3.70 & 11.11 & MR & 3.70 & 7.41 & $\mathrm{R}$ \\
\hline 2 & DCA 69 & 0.00 & 7.40 & $\mathrm{R}$ & 0.00 & 7.41 & $\mathrm{R}$ & 0.00 & 3.70 & $\mathrm{R}$ \\
\hline 3 & DCA 76 & 3.70 & 7.41 & $\mathrm{R}$ & 7.41 & 25.93 & MR & 3.70 & 18.52 & MR \\
\hline 4 & DCA 79 & 0.00 & 3.70 & $\mathrm{R}$ & 0.00 & 18.52 & MR & 0.00 & 40.74 & MS \\
\hline 5 & DCA 81 & 0.00 & 7.41 & $\mathrm{R}$ & 0.00 & 33.33 & MS & 3.70 & 40.74 & MS \\
\hline 6 & DCA 89 & 0.00 & 3.70 & $\mathrm{R}$ & 0.00 & 7.41 & $\mathrm{R}$ & 0.00 & 7.41 & $\mathrm{R}$ \\
\hline 7 & DCA 98 & 0.00 & 11.11 & MR & 3.70 & 33.33 & MS & 0.00 & 18.52 & MR \\
\hline 8 & DCA 104 & 0.00 & 7.41 & $\mathrm{R}$ & 0.00 & 25.93 & MR & 0.00 & 25.93 & MR \\
\hline 9 & DCA 107 & 7.41 & 55.56 & $S$ & 7.41 & 25.93 & MR & 3.70 & 18.52 & MR \\
\hline 10 & DCA 118 & 0.00 & 3.70 & $\mathrm{R}$ & 0.00 & 33.33 & MS & 7.41 & 25.93 & MR \\
\hline 11 & DCA 123 & 0.00 & 7.41 & $\mathrm{R}$ & 3.70 & 25.93 & MR & 7.41 & 25.93 & MR \\
\hline 12 & DCA 145 & 11.11 & 62.96 & $\mathrm{~S}$ & 0.00 & 7.41 & $\mathrm{R}$ & 0.00 & 25.93 & MR \\
\hline 13 & DCA 153 & 0.00 & 7.41 & $\mathrm{R}$ & 0.00 & 11.11 & MR & 0.00 & 18.52 & MR \\
\hline 14 & DCA 154 & 0.00 & 11.11 & MR & 3.70 & 25.93 & MR & 0.00 & 11.11 & MR \\
\hline 15 & DCA 174 & 0.00 & 7.41 & $\mathrm{R}$ & 7.41 & 40.74 & MS & 7.41 & 55.56 & S \\
\hline 16 & DCA 176 & 0.00 & 11.11 & MR & 3.70 & 18.52 & MR & 3.70 & 25.93 & MR \\
\hline 17 & DCA 189 & 0.00 & 3.70 & $\mathrm{R}$ & 3.70 & 7.41 & $\mathrm{R}$ & 0.00 & 7.41 & $\mathrm{R}$ \\
\hline 18 & DCA 216 & 0.00 & 3.70 & $\mathrm{R}$ & 0.00 & 33.33 & MS & 0.00 & 18.52 & MR \\
\hline 19 & DCA 222 & 3.70 & 11.11 & MR & 3.70 & 11.11 & MR & 0.00 & 11.11 & MR \\
\hline 20 & DCA 224 & 0.00 & 7.41 & $\mathrm{R}$ & 3.70 & 11.11 & MR & 0.00 & 25.93 & MR \\
\hline 21 & DCA 233 & 0.00 & 7.41 & $\mathrm{R}$ & 7.41 & 33.33 & MS & 7.41 & 33.33 & MS \\
\hline 22 & DCA 234 & 7.41 & 33.33 & MS & 11.11 & 33.33 & MS & 0.00 & 18.52 & MR \\
\hline 23 & DCA 237 & 0.00 & 3.70 & $\mathrm{R}$ & 3.70 & 40.74 & MS & 3.70 & 33.33 & MS \\
\hline 24 & DCA 240 & 3.70 & 11.11 & MR & 0.00 & 25.93 & MR & 3.70 & 25.93 & MR \\
\hline 25 & DCA 257 & 0.00 & 18.52 & MR & 3.70 & 18.52 & MR & 0.00 & 33.33 & MS \\
\hline 26 & DCA 295 & 0.00 & 18.52 & MR & 11.11 & 25.93 & MR & 0.00 & 25.93 & MR \\
\hline 27 & DCA 298 & 11.11 & 55.56 & $S$ & 3.70 & 55.56 & $\mathrm{~S}$ & 7.41 & 11.11 & MR \\
\hline 28 & DCA 300 & 0.00 & 7.41 & $\mathrm{R}$ & 7.41 & 11.11 & MR & 0.00 & 33.33 & MS \\
\hline 29 & DCA 302 & 0.00 & 33.33 & MS & 0.00 & 11.11 & MR & 0.00 & 7.41 & $\mathrm{R}$ \\
\hline 30 & DCA 305 & 0.00 & 7.41 & $\mathrm{R}$ & 0.00 & 25.93 & MR & 3.70 & 11.11 & MR \\
\hline 31 & ByadagiDabbi & 0.00 & 18.52 & MR & 11.11 & 25.93 & MR & 3.70 & 55.56 & $\mathrm{~S}$ \\
\hline
\end{tabular}


Table.3 Reaction of chilli genotypes under natural condition, challenge inoculation and invitro fruit inoculation

\begin{tabular}{|c|c|c|c|c|c|}
\hline & Resistant & $\begin{array}{l}\text { Moderately } \\
\text { resistant }\end{array}$ & $\begin{array}{l}\text { Moderately } \\
\text { susceptible }\end{array}$ & Susceptible & $\begin{array}{l}\text { Highly } \\
\text { susceptible }\end{array}$ \\
\hline \multicolumn{6}{|c|}{ Screening under natural condition } \\
\hline 90 DAT & $\begin{array}{l}\text { DCA-67, } \\
69,76,189, \\
237,257, \\
302 \text { and } 305\end{array}$ & $\begin{array}{l}\text { DCA-79, 81, 89, } \\
98,104,107, \\
118,123,145, \\
153,154,174, \\
176,216,222, \\
224,233,234, \\
240,295,298, \\
300 \text { and } \\
\text { ByadagiDabbi }\end{array}$ & & & \\
\hline $\begin{array}{l}\text { During } \\
\text { harvest }\end{array}$ & $\begin{array}{l}\text { DCA-69 } \\
\text { and } 189\end{array}$ & $\begin{array}{l}\text { DCA-67, } 76,89, \\
123,237,300, \\
302 \text { and } 305\end{array}$ & $\begin{array}{l}67,79,81,98, \\
104,107,118, \\
145,153,154, \\
174,176,216, \\
222,224,233, \\
234,240,257, \\
295 \text { and } 298\end{array}$ & ByadagiDabbi & \\
\hline \multicolumn{6}{|c|}{ In vitro fruit inoculation } \\
\hline $\begin{array}{l}\text { Green } \\
\text { stage }\end{array}$ & $\begin{array}{l}\text { DCA-67, } \\
69,76,79, \\
81,89,104, \\
118,123, \\
153,174, \\
189,216, \\
224,233, \\
237,300 \\
\text { and } 305 .\end{array}$ & $\begin{array}{l}\text { DCA-98, 154, } \\
\text { 176, 222, 240, } \\
257,295 \text { and } \\
\text { ByadagiDabbi. }\end{array}$ & $\begin{array}{l}\text { DCA-234 and } \\
302\end{array}$ & $\begin{array}{l}\text { DCA-107, } 145 \\
\text { and } 298 .\end{array}$ & \\
\hline $\begin{array}{l}\text { Turning } \\
\text { red stage }\end{array}$ & $\begin{array}{l}\text { DCA-69, } \\
89,145 \text { and } \\
189\end{array}$ & $\begin{array}{l}\text { DCA-67, 76, 79, } \\
104,107,123, \\
153,154,176, \\
222,224,240, \\
257,295,300, \\
302,305 \text { and } \\
\text { ByadagiDabbi }\end{array}$ & $\begin{array}{l}\text { DCA- } 81,98, \\
118,174,216, \\
233,234 \text { and } \\
237 .\end{array}$ & DCA-298. & \\
\hline $\begin{array}{l}\text { Red ripe } \\
\text { stage }\end{array}$ & $\begin{array}{l}\text { DCA-67, } \\
69,89,189 \\
\text { and } 302\end{array}$ & $\begin{array}{l}\text { DCA-76, 98, } \\
104,107,118, \\
123,145,153, \\
154,176,216, \\
222,224,234, \\
240,295,298 \\
\text { and } 305 .\end{array}$ & $\begin{array}{l}\text { DCA-79, } 81, \\
233,237,257 \\
\text { and } 300 .\end{array}$ & $\begin{array}{l}\text { DCA- } 174 \text { and } \\
\text { ByadagiDabbi. }\end{array}$ & \\
\hline
\end{tabular}


Fig.1 In vitro screening of different entries of chilli at green stage
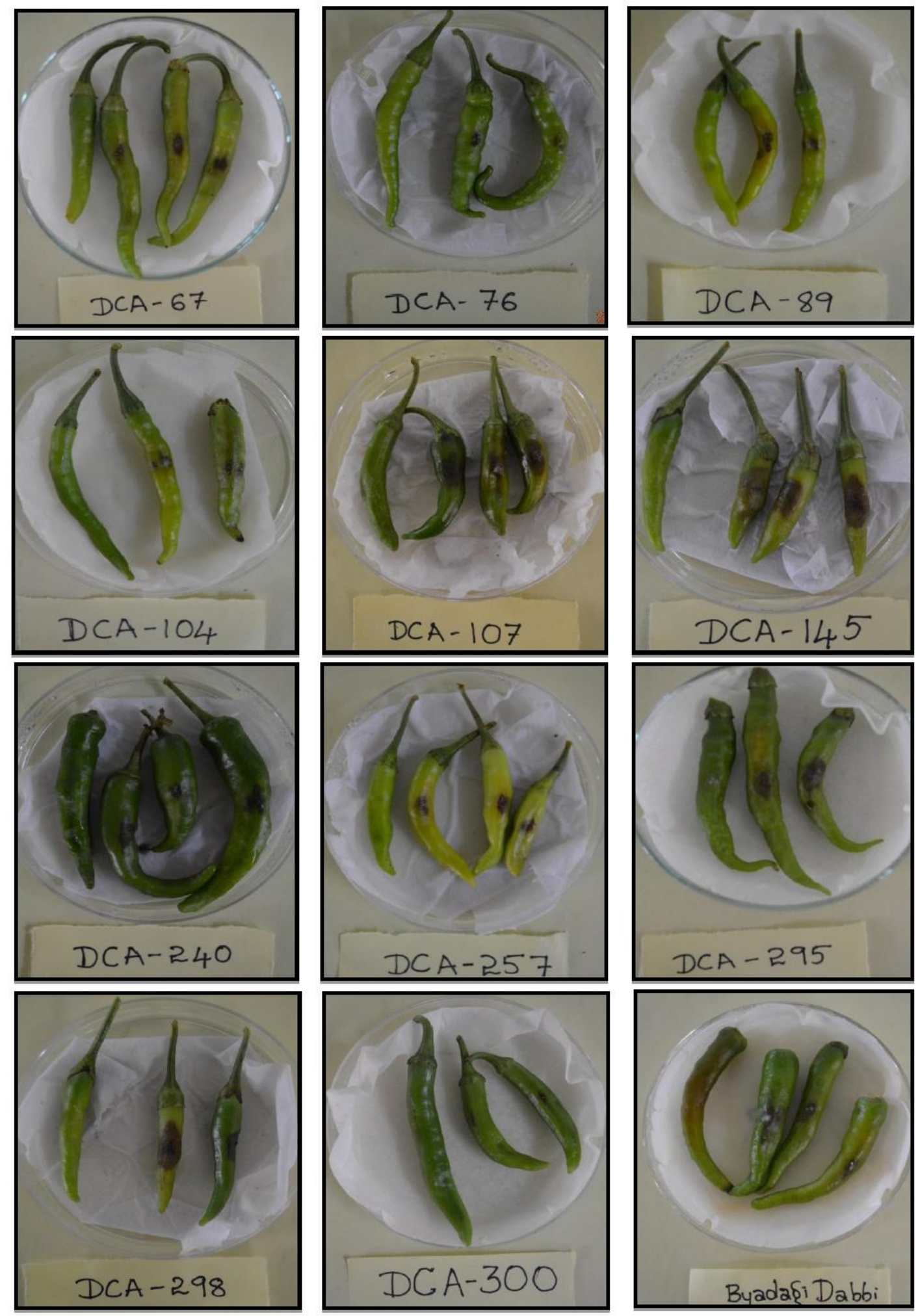
Plate.2 In vitro screening of different entries of chilli at red ripe stage
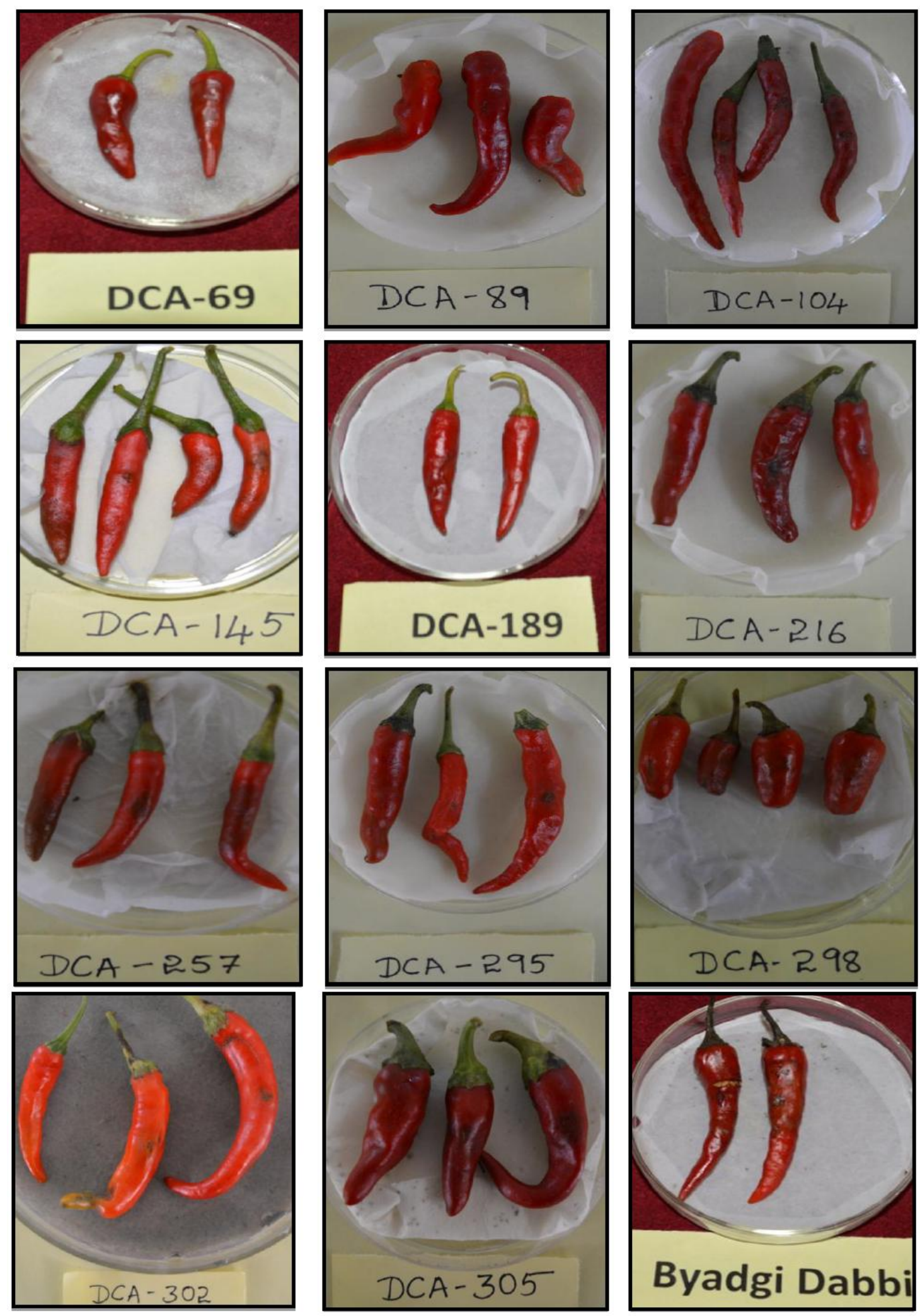
In vitro screening of 31 chilli accessions, 18accessions were found resistant, 8 were moderately resistant, 3 were moderately susceptible and 2 were susceptible to the pathogen during green stage of the fruit. The reaction of chilli fruits during turningred stage recorded, 18accessionsas moderately resistant, 8 as moderately susceptible and one (DCA-298) was susceptible. The reaction varied in red stage of fruit development wherein, 5accessionswere resistant, 18accessions were moderately resistant, 6 were moderately susceptible and 2 were susceptible to the pathogen. The pathogen infection was more in case of red stages of the fruit development as compared to mature green stage. Many accessions were found resistant during green stages, but susceptible at red stages of the fruit development. Few accessions were tolerant to the pathogen at red stage but susceptible during green stages. Mesta (1996) found 11 accessions as resistant and 24 as moderately resistant accessions against Colletotrichum capsici out of 217 chilli accessions screened under natural and lab condition.

The accessions DCA-67, 69, 189, 302 and 305 were reacted resistant to the $C$. capsici in field and artificial screening. Hence, these accessions having the resistant genes which can be harnessed for resistant breeding programme.

\section{References}

Anonymous, 2018, Indian Horticulture Database-2018. National Horticulture Board., 139-140.

Bosland, P. W., 1994, Chiles: history, cultivation, and uses, in Spices, Herbs, and Edible Fungi, Charalambous, G., (Ed) Elsevier Publishing, New York, pp. 347-366.

Kaur, D. and Singh, D., 2009, Evaluation of hot pepper (Capsicum annuum L.) germplasm of diverse origin for various horticultural characters.Indian J. Plant Genet.Resour.,22(1): 41-45.

Koppad, S. R., 2014, Studies on ripe fruit rot of chilli caused by Colletotrichum capsici (Sydow) Butler and Bisby with special reference to biochemical basis of resistance. M. Sc. Thesis, Univ.Horti.Sci., Bagalkot (India).

Mayee, C. D. and Datar, V. V., 1986, Phytopathometry, Technical bulletin1.Marathwada Agric. Univ., Parbhani, pp: 46.

Mesta, R. K., 1996, Studies on fruit rot of chilli (Capsicum annum L.) caused by Colletotrichum capsici (Sydow) Butler and Bisby, M. Sc. (Agri.) Thesis, Univ. Agric. Sci., Dharwad (India).

Perry, L., Dickau, R., Zarrillo, S., Holst, I., Pearsall, D. M., Piperno, D. R., Berman, M. J., Cooke, R. G., Rademaker, K. and Ranere, A. J., 2007, Starch fossils and the domestication and dispersal of chili peppers (Capsicum spp. L.) in the Americas. Science.,315: 986-988.

Prasath, D. and Ponnuswami, V., 2008, Heterosis and combining ability for morphological, yield and quality characters in paprika type chilli hybrids.Indian J. Hortic., 65: 441-445.

Ridzuan, R., Rafii, M. Y., Ismail, S. I., Mohammad Yusoff, M., Miah, G. and Usman, M., 2018, Breeding for anthracnose disease resistance in chili: progress and prospects. International journal of molecular sciences., 19(10): 3122.

Ruth, B. R. A., Veeraraghavathatham, D. and Prakasam, V., 2007, Screening of chilli germplasm for anthracnose resistance.Madras Agric. J., 94(1-6): 14-22.

Singh, R. and Chowdhury, A. K., 2011, Evaluation of chilli germplasms against anthracnose (Colletotrichum capsici) 
under field conditions. Journal of research, SKUAST-J., 10(1): 100-104.

Varma, K. N., Anitha, K., Kumar, G. S., Pandravada, S. R., Satyanarayana, J., Prasadini, P. P. and Reddy, D. J., 2012, Identification of resistant sources against Colletotrichum capsici in
Capsicum germplasm.Indian Journal of Plant Protection.,40 (3): 230-236.

Wheeler, B. E. J., 1969, An introduction to plant diseases. John Wiley and Sons Ltd., London, pp. 301.

\section{How to cite this article:}

Sharath, M. N., R. K. Mesta, M. A. Kareem, P. S. Ajjappalavara and Ambresh. 2020. Screening of Chilli Genotypes for Resistance to Fruit Rot caused by Colletotrichum capsici at Fruit Bearing and Harvest Stages. Int.J.Curr.Microbiol.App.Sci. 9(08): 142-152.

doi: https://doi.org/10.20546/ijcmas.2020.908.015 